\title{
Plasma core power exhaust in ELMy H-Mode in JET with ITER-Like Wall
}

\author{
C. Guillemaut ${ }^{1,2}$, C. Metzger ${ }^{1,3}$, L. Appel' ${ }^{4}$, P. Drewelow ${ }^{5}$, L. Horvath ${ }^{6}$,
} G. Matthews ${ }^{4}$, G. Szepesi ${ }^{4}$, E. Solano ${ }^{7}$, and JET contributors*

\author{
${ }^{1}$ EUROfusion Consortium, JET, Culham Science Centre, Abingdon, OX14 3DB, UK \\ ${ }^{2}$ Instituto de Plasmas e Fusão Nuclear, Instituto Superior Técnico, Universidade Lisboa, PT \\ ${ }^{3}$ Ambassade de France à Londres, 21 Cromwell Road, London SW7 2EN, UK \\ ${ }^{4}$ CCFE, Culham Science Centre, Abingdon OX14 3DB, UK \\ ${ }^{5}$ Max-Planck-Institut fur Plasmaphysik, Teilinstitut Greifswald, D-17491 Greifswald, Germany \\ ${ }^{6}$ YPI, University of York, York YO10 5DQ, UK \\ 7 Laboratorio Nacional de Fusión, CIEMAT, 28040 Madrid, Spain \\ "See the author list of "Litaudon et al, Nucl. Fusion 57 (2017) 102001" \\ Contact Email: christophe.guillemaut@ukaea.uk or cguillemaut@ipfn.ist.utl.pt
}

\begin{abstract}
The mitigation of target heat load in future steady state fusion devices will require dissipation of a significant amount of power through radiation. Plasma operations relying on ELMy $\mathrm{H}$-modes could be problematic since ELMs may transport substantial amounts of power to the target without significant dissipation. Therefore, estimation of the average ELM power exhaust from the plasma core is crucial to evaluate the potential limitation on the power dissipation in ELMy $\mathrm{H}$-mode regime. A series of more than 50 JET with ITER-Like Wall (JET-ILW) H-mode discharges with a wide range of conditions has been used here to compare the average ELM power to the average input power. The effect of input power, ELM frequency, plasma current, confinement and radiation on ELM power exhaust has been studied and reported in this paper. Good agreement has been found here with previous studies made in carbon machines. This work suggests that it should not be possible to dissipate more than $70-80 \%$ of the input power in ELMy H-modes in JET-ILW which is consistent with the maximum radiative fraction found experimentally.
\end{abstract}

\section{Introduction}

The present actively cooled plasma facing components cannot cope with power densities greater than $\sim 10 \mathrm{MW} \cdot \mathrm{m}^{-2}$ in steady state. Safe operation of future fusion devices with such technology will require dissipation of large amounts of power through radiation mostly. ITER should require $60-70 \%$ of exhaust power dissipation [1] and DEMO may need more than $90 \%$ [2] for baseline steady state operations. In this context, plasma operations relying on ELMy $\mathrm{H}$-mode plasmas could be problematic since Edge-Localized Modes (ELMs) may transport a substantial fraction of the exhaust power without significant dissipation. Indeed, ELMy ions seem to carry most of the ELM energy and can reach multi-keV levels [3-5]. At these energies, the ion collisionality is expected to be very low [6] which would render the use of radiative impurity seeding essentially ineffective during ELMs.

Estimation of the average ELM power exhaust $\left(\left\langle P_{E L M}\right\rangle\right)$ from the plasma core is crucial to evaluate the potential limitation on power dissipation in ELMy $\mathrm{H}$-mode discharges. It is known from previous studies on JET-Carbon (C) [7], DIII-D [8] and ASDEX-Upgrade with C wall [9] that: 
$\left\langle P_{E L M}\right\rangle \approx f_{E L M} \times \Delta W_{E L M} \approx 0.3-0.4\left\langle P_{\text {in }}\right\rangle$,

with the ELM frequency $f_{E L M}$ in $\mathrm{Hz}$, the ELM energy loss $\triangle W_{E L M}$ in $\mathrm{J}$ and the average total input power $\left\langle P_{i n}\right\rangle$ in $\mathrm{W}$. In present machines, in absence of significant fusion power, $\left\langle P_{i n}\right\rangle$ represents the average exhaust power.

The objective of the study presented here is to verify if relation (1) is still valid in JET with ITER-Like Wall (JET-ILW) comprising a tungsten divertor with a beryllium (Be) main chamber wall as ITER, in order to evaluate the potential limitation on exhaust power dissipation. For this purpose, a data set of 51 JET-ILW Type-I ELMy $\mathrm{H}$-mode discharges with a very wide range of ELM frequency $\left(f_{E L M}\right),\left\langle P_{i n}\right\rangle$, plasma current $\left(I_{p}\right)$, toroidal field and pedestal conditions have been used. These discharges were achieved with deuterium or hydrogen as main species and a few of them involved nitrogen $(\mathrm{N})$ or neon $(\mathrm{Ne})$ seeding. The configuration in use in this series of experiments features a vertical inner target and a horizontal outer target, see Fig. 1.

The measurement method of the ELM energy loss is described in Section 2. The dependence of $\left\langle P_{E L M}\right\rangle$ on $\left\langle P_{i n}\right\rangle, f_{E L M}, I_{p}$ and the confinement ( $H_{98}$ factor) is presented in Section 3 and finally, before concluding, the possible limitation on the radiative power fraction in JET-ILW is discussed in Section 4.

\section{Measurement of ELM energy losses}

In JET-ILW, EFIT equilibrium reconstructions use measurements from arrays of flux loops, saddle loops and pick up coils placed all around the plasma chamber, see Fig. $1 \mathrm{a}$ and $\mathrm{b}$. The plasma stored energy $W_{P}$ is obtained by volume integration of the pressure profile from EFIT. This method is considered reliable since comparison with volume integration from Thomson Scattering pressure profiles yield similar values of $W_{P}[10]$.

$\triangle W_{E L M}$ can be estimated by measuring the variation of $W_{P}$ during ELMs. A coherent averaging method [11] using Be II spectroscopy (Fig. 1b) as an ELM marker has been used to obtain a typical average ELM time trace for each of the 51 cases studied here. The coherently averaged $W_{P}$ signal obtained with this method for the Type-I ELM example from discharges \#84584 is shown in Fig. 2.

It has been found that Type-I ELMy discharges with $f_{E L M}$ up to $\sim 100 \mathrm{~Hz}$ tend to show a time trace of $W_{P}$ like in \#84584 with an abrupt crash synchronized with the ELM. The measurement of $\triangle W_{E L M}$ consist in calculating the variation between the maximum and minimum of $W_{P}$ before and after the ELM, respectively.

For smaller and faster ELMs a $\triangle W_{E L M}$ can also be calculated but the crash of $W_{P}$ is not synchronized with the ELM. Fast changes in the plasma during ELMs can induce currents in the surrounding passive structures of the machine and generate magnetic perturbation which will be picked up by the different coils and loops with a delay. The faster the change, the stronger the induced current. Therefore, the time shifted $\triangle W_{E L M}$ measurements are likely to be affected by this effect and have been discarded in this study. Only the $\triangle W_{E L M}$ data from $W_{P}$ crashes synchronized with ELMs is considered fully reliable.

Mention should be made that in this study, $\triangle W_{E L M}$ will only be compared to $\left\langle P_{i n}\right\rangle$ without considering the power balance issue in JET-ILW [12]. 


\section{ELM power exhaust in JET-ILW}

The $\Delta W_{E L M}$ measurements allow the calculation of $\left\langle P_{E L M}\right\rangle$ with equation (1) for the different discharges studied here. The possible dependences of $\left\langle P_{E L M}\right\rangle /\left\langle P_{i n}\right\rangle$ on $\left\langle P_{i n}\right\rangle$, $f_{E L M}$, the $H_{98}$ factor and $I_{p}$ have been investigated and are shown in Fig. 3a, b, c and d, respectively.

It can already be concluded that $\left\langle P_{E L M}\right\rangle /\left\langle P_{i n}\right\rangle$ does not show any clear dependence on $\left\langle P_{i n}\right\rangle, f_{E L M}, I_{p}$ or the $H_{98}$ factor. The values for the different cases studied here are scattered between 0.2 and 0.5 around a rather constant average of $\sim 0.35$ in any conditions as in previous studies in C machines [7-9].

In Fig. 4, the average core radiative power $\left(\left\langle P_{\text {rad core }}\right\rangle\right)$ measured by bolometry (Fig. 1c) has been subtracted to $\left\langle P_{i n}\right\rangle$ to estimate $\left\langle P_{E L M}\right\rangle$ independently of the core radiative losses. In this case, most of the measurements verify the following relation:

$\left\langle P_{E L M}\right\rangle \approx f_{E L M} \times \Delta W_{E L M} \approx 0.4\left(\left\langle P_{\text {in }}\right\rangle-\left\langle P_{\text {rad core }}\right\rangle\right)$,

also independently of $\left\langle P_{i n}\right\rangle, f_{E L M}, I_{p}$ or the $H_{98}$ factor. The data is scattered between 0.2 and 0.6. The small difference between relation (1) and (2) suggests that the core does not radiate a lot of power in JET-ILW. When $\mathrm{N}$ or Ne seeding is involved, the radiative power is mostly produced in low temperature regions in the divertor.

More generally, it is worth noting that ELMs always exhaust a minimum of $20-$ $30 \%$ of $\left\langle P_{i n}\right\rangle$ in JET-ILW which means that not more than $70-80 \%$ of $\left\langle P_{i n}\right\rangle$ flows out of the core into the scrape-off layer during inter-ELM.

\section{Discussion on the radiative power fraction limitation in JET-ILW}

Coherent averaging of total radiative power measured by bolometry is shown on Fig. 5 for the Type-I ELMs of \#84584. The low-path filter used to amplify the bolometer signals introduce a delay of $\sim 2.5 \mathrm{~ms}$ in the measurements while the delay observed between Be II spectroscopy and bolometry in Fig. 5 is of the order of $\sim 4-5 \mathrm{~ms}$. Therefore, it is unlikely that the delayed radiative power spike associated with the ELM is due to dissipation of the ELM power.

Time integration of the radiative power above the pre-ELM steady level (pink area in Fig. 5) yields a radiated energy of $\approx 7 \mathrm{~kJ}$ for \#84584 while $\Delta W_{E L M} \approx 230 \mathrm{~kJ}$ in this case. If the decrease of radiative power below the pre-ELM steady level (blue area in Fig. 5) is accounted for, the integration yield nearly zero which means that in average ELMs do not add any contribution to the inter-ELM level of radiation in discharge \#84584. The transient reduction of radiative power below the steady preELM level is likely to be due to the reduction of pedestal density during the ELM. Such small amounts of radiated energy is likely to come from some form of recycling.

It is shown in [13] that the seeding of radiative impurities increases the amount of radiated energy above the pre-ELM steady level without further reduction after the spike. However, the same delay is observed between the ELM as seen by Be II spectroscopy and the associated radiative power measured by bolometry. Therefore, the use of radiative impurity essentially enhances the inter-ELM power dissipation without dissipating the ELM energy itself. 
This is consistent with the "Free-Streaming" kinetic model (FSM) which describes ELMs as a plasma bunch expanding in vacuum along the magnetic field lines $[6,14,15]$. The model is based on the assumptions that ELMs do not interact with the plasma background and conserve quasi-neutrality during their parallel transport from pedestal to targets. The FSM predicts that in JET-ILW, the maximum target ion impact energy $\left(E_{i, \text { max }}\right)$ should be such that:

$$
E_{i, \text { max }} \approx 5.23 T_{e, \text { max }}^{\text {ped }} \text {, }
$$

with $T_{e, \max }^{p e d}$ the maximum pedestal electron temperature before the ELM crash.

This has been verified for the 51 Type-I ELMy H-mode discharges studied here and also for 31 other cases containing faster Type-I ELMs as well as Type-III ELMs [5]. Estimations of $E_{i, \max }$ from outer target Langmuir probes (LPs) and Infrared thermography (IR) measurements (Fig. 1d) plotted against $T_{e, \text { max }}^{p e d}$ measurements made with Electron Cyclotron Emission (ECE) show very good agreement with the FSM prediction in Fig. 5. The conservation of quasi-neutrality forces the electrons to transfer most of their parallel energy to the ions which become essentially kinetic with a dominant parallel motion and reach multi-keV energies.

LP measurements of electron temperature during ELMs $\left(T_{e, E L M}\right)$ allow calculation of the electron impact energy $\left(E_{e}\right)$ as follows:

$$
E_{e} \approx \gamma_{e} T_{e, E L M}
$$

with the electron heat transmission coefficient $\gamma_{e} \approx 5.5$ [16]. As shown in Fig. 5, $E_{e}$ estimations for the 82 cases considered are always very small compared to $E_{i, \max }$, as expected from the FSM.

Such experimental validation of the FSM suggests that the assumption that ELMy ions do not interact with the plasma background is true for Type-I and Type-III ELMs. Multi-keV ions will indeed have a very low collisionality and are unlikely to dissipate their energy before reaching the target. This is consistent with a previous study based on a fluid model for ELMs [17]. Since in JET-ILW at least $20-30 \%$ of $\left\langle P_{i n}\right\rangle$ is carried out of the core through this channel, not more than $70-80 \%$ of $\left\langle P_{i n}\right\rangle$ can be radiated in ELMy $\mathrm{H}$-mode. This could explain the radiative fraction limit found on JET-ILW in seeded ELMy H-modes [18]. This level of power dissipation should be sufficient for ITER but not enough for DEMO.

\section{Conclusions}

Safe operation of future fusion devices with present technology of actively cooled plasma facing components will require dissipation of large amounts of power through radiation. ELMy $\mathrm{H}$-mode plasmas could be problematic since Edge-Localized Modes (ELMs) may transport a substantial fraction of the exhaust power without dissipation. Estimation of the average ELM power exhaust $\left(\left\langle P_{E L M}\right\rangle\right)$ from the plasma core has been carried out in 51 JET with ITER-Like Wall (JET-ILW) ELMy H-mode discharges to evaluate the potential limitation on power dissipation.

The study presented in this paper has confirmed that $\left\langle P_{E L M}\right\rangle$ represents $\sim 35$ $\%$ of the average input power $\left(\left\langle P_{i n}\right\rangle\right)$ in JET-ILW with a scatter between $20 \%$ and 50 
$\%$ as in previous studies on carbon machines [7-9]. As previously, this fraction appears to be independent of $\left\langle P_{i n}\right\rangle$, the ELM frequency, the plasma current or the confinement. If the average core radiative power is subtracted from $\left\langle P_{i n}\right\rangle,\left\langle P_{E L M}\right\rangle$ represents $\sim 40 \%$ of the available core input power also independently of all the quantities tested here.

Inspection of coherently averaged bolometry measurements reveals that the spikes of total radiative power associated with ELMs occur essentially after the ELMs. Therefore, the spikes cannot be due to the dissipation of the ELM power itself but are likely to be related to some form of recycling. As already discussed in [13], the use of impurity seeding in ELMy $\mathrm{H}$-mode only enhances the radiative dissipation during inter-ELM.

The validation of the free-streaming nature of ELMy particles [3-5] which implies no interactions with the plasma background is consistent with the fact that $\left\langle P_{E L M}\right\rangle$ cannot be dissipated before the target. As a result, not more than $70-80 \%$ of $\left\langle P_{i n}\right\rangle$ can be radiated in JET-ILW ELMy $\mathrm{H}$-modes which is consistent with the experimental limit found in [18]. This fraction of exhaust power dissipation would be sufficient for ITER but not enough for DEMO.

\section{Acknowledgments}

This work has been carried out within the framework of the EUROfusion Consortium and has received funding from the Euratom research and training programme 20142018 under grant agreement No 633053. IST activities also received financial support from "Fundação para a Ciência e Tecnologia" through project UID/FIS/50010/2013. The views and opinions expressed herein do not necessarily reflect those of the IST or of the European Commission.

\section{References}

[1] R.A. Pitts et al., Phys. Scr. T138 (2009) 014001.

[2] G. Federici et al., Fusion Eng. Des. 89 (2014) 882-889.

[3] C. Guillemaut et al., Phys. Scr. T167 (2016) 014005.

[4] C. Guillemaut et al., Plasma Phys. Control. Fusion 57 (2015) 085006

[5] C. Guillemaut et al., submitted to be published in Nucl. Fusion

[6] D. Moulton et al., Plasma Phys. Control. Fusion 55 (2013) 085003

[7] A. Loarte et al., Plasma Phys. Control. Fusion 44 (2002) 1815

[8] A. Leonard et al., J. Nucl. Mater. 266-269 (1999) 109

[9] W. Suttrop et al., Plasma Phys. Control. Fusion 42 (2000) A97

[10] L. Horvath et al., http://ocs.ciemat.es/EPS2017PAP/pdf/P5.123.pdf

[11] R.A. Pitts et al., Nucl. Fusion 43 (2003) 1145-1166

[12] G.F. Matthews et al., http://dx.doi.org/10.1016/i.nme.2016.12.012

[13] A.R. Field et al., Plasma Phys. Control. Fusion 59 (2017) 095003

[14] W. Fundamenski et al., Plasma Phys. Control. Fusion 48 (2006) 109-156

[15] T. Eich et al., J. Nucl. Mater. 390-391 (2009) 760-763

[16] P.C. Stangeby 2000, The Plasma Boundary of Magnetic Fusion Devices (New York: Taylor and Francis)

[17] J. Rapp et al., Nucl. Fusion 44 (2004) 312-319

[18] M. Wischmeier et al., J. Nucl. Mater. 463 (2015) 22-29 


\section{Figures captions:}

Fig. 1 Diagnostics and examples of the plasma configurations used in this study. a) Saddle loops, b) Da spectroscopy, Electron Cyclotron Emission (ECE), flux loops and pick up coils, c) bolometry lines of sights and d) divertor Infrared thermography (IR).

Fig. 2 Coherent averaging of $W_{P}$ (magenta bullets) and Be II spectroscopy (blue bullets) signals for discharge \#84584 (Type-I ELMs).

Fig. 3 Normalized $\left\langle P_{E L M}\right\rangle$ against a) Pin, b) $f_{E L M}$, c) $H_{98}$ and d) $I_{p \text {. }}$.

Fig. 4 As in Fig. 3 but $\left\langle P_{E L M}\right\rangle$ is now normalized to $\left(\left\langle P_{\text {in }}\right\rangle-\left\langle P_{\text {rad core }}\right\rangle\right)$.

Fig. 5 Coherent averaging of total radiative power from bolometry (red bullets) and Be II spectroscopy (blue bullets) signals for discharge \#84584 (Type-I ELMs).

Fig. 6 Linear dependence between $E_{i, \text { max }}$ and $T_{e, \text { max }}^{\text {ped }}$ during ELMs (red and blue bullets). No dependence can be found between $E_{e}$ and $T_{e}^{\text {ped }}$ (red and blue squares). Red points correspond to Type-I ELMs experimental data and blue points to Type-III ELMs. The black line equation is $y=5.23 x$. 


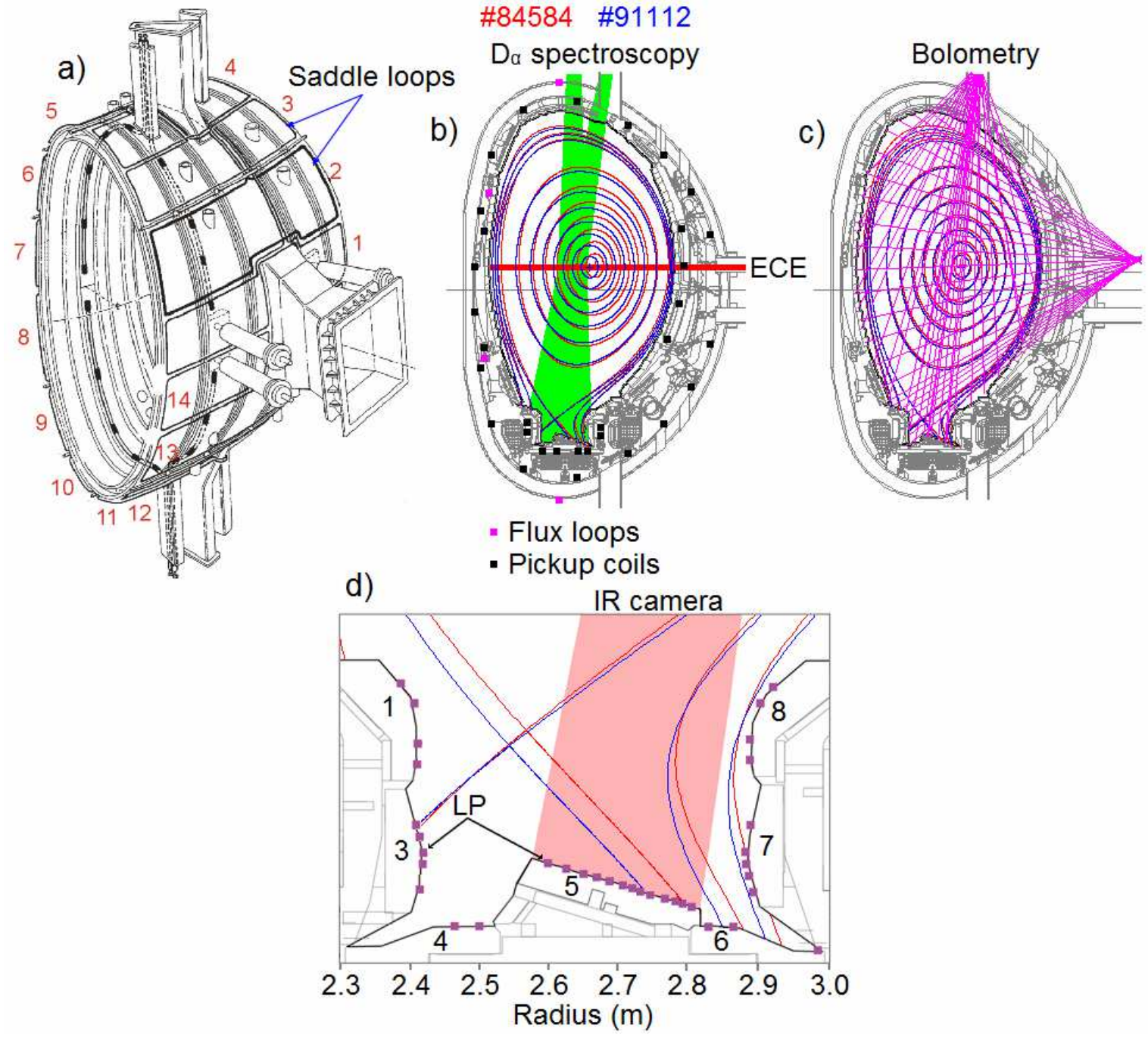

Fig. 1 


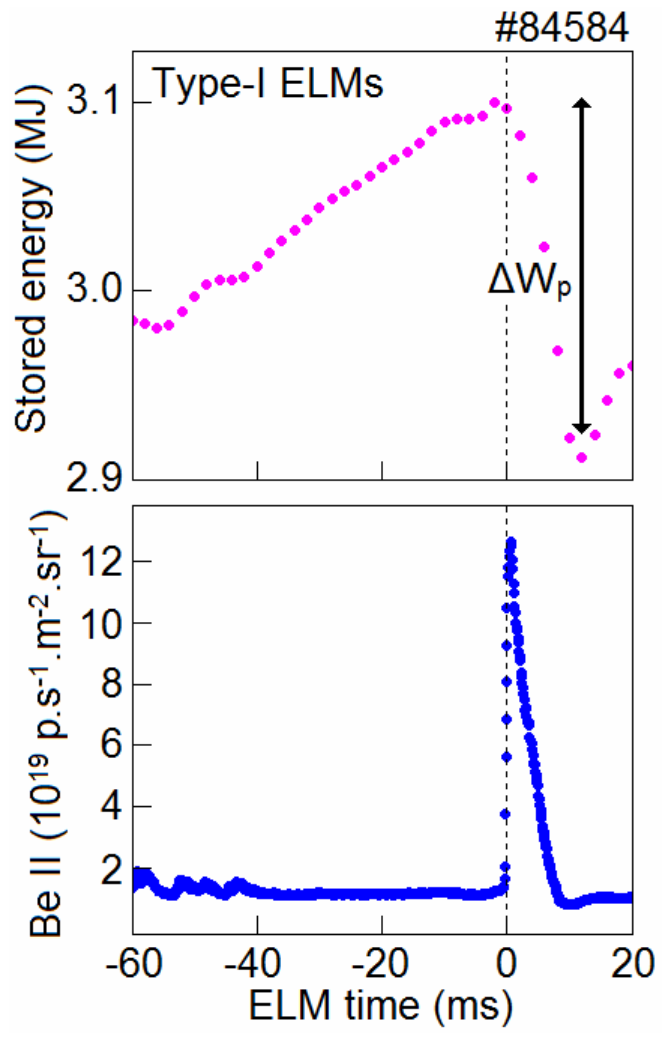

Fig. 2 

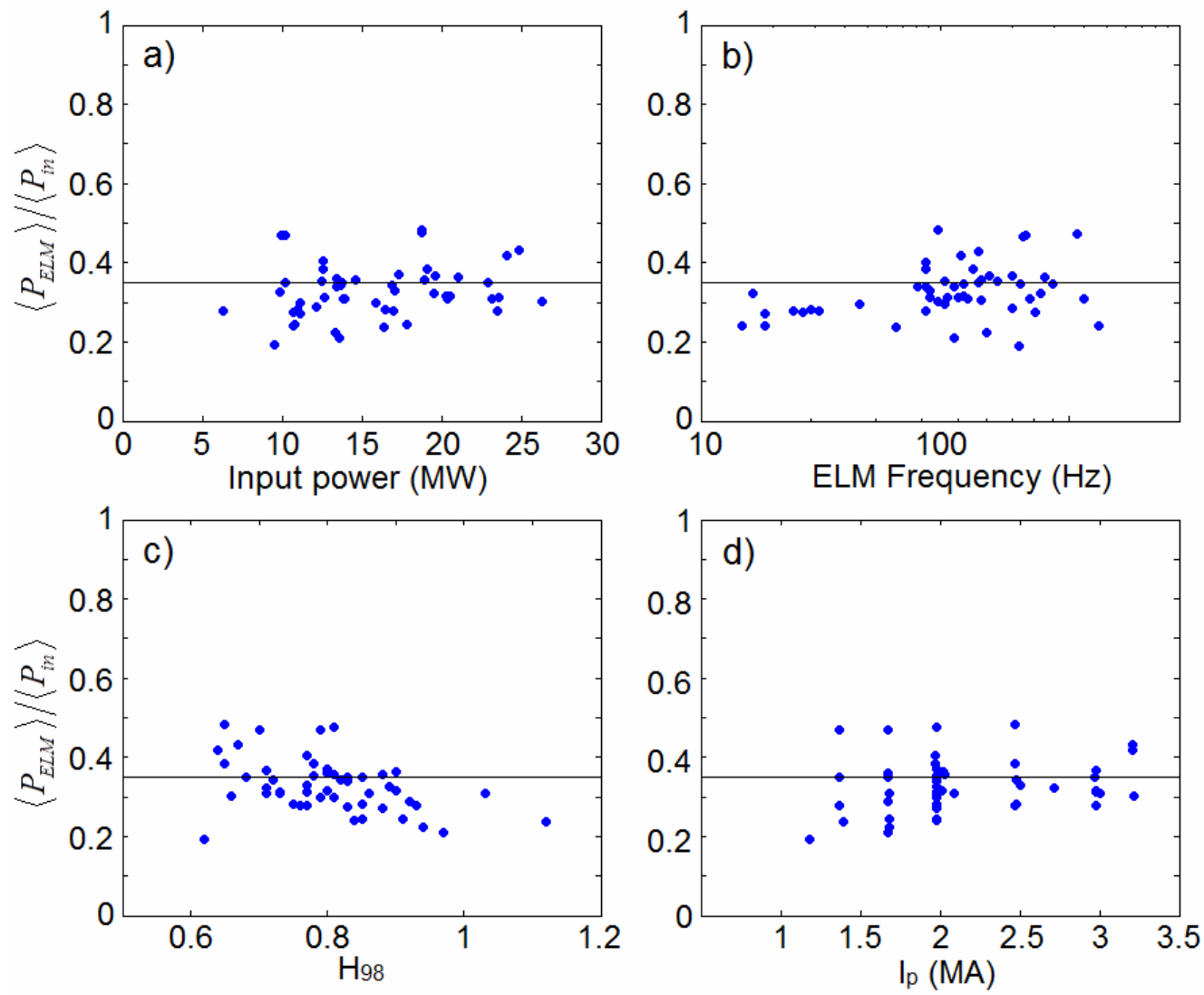

Fig. 3 

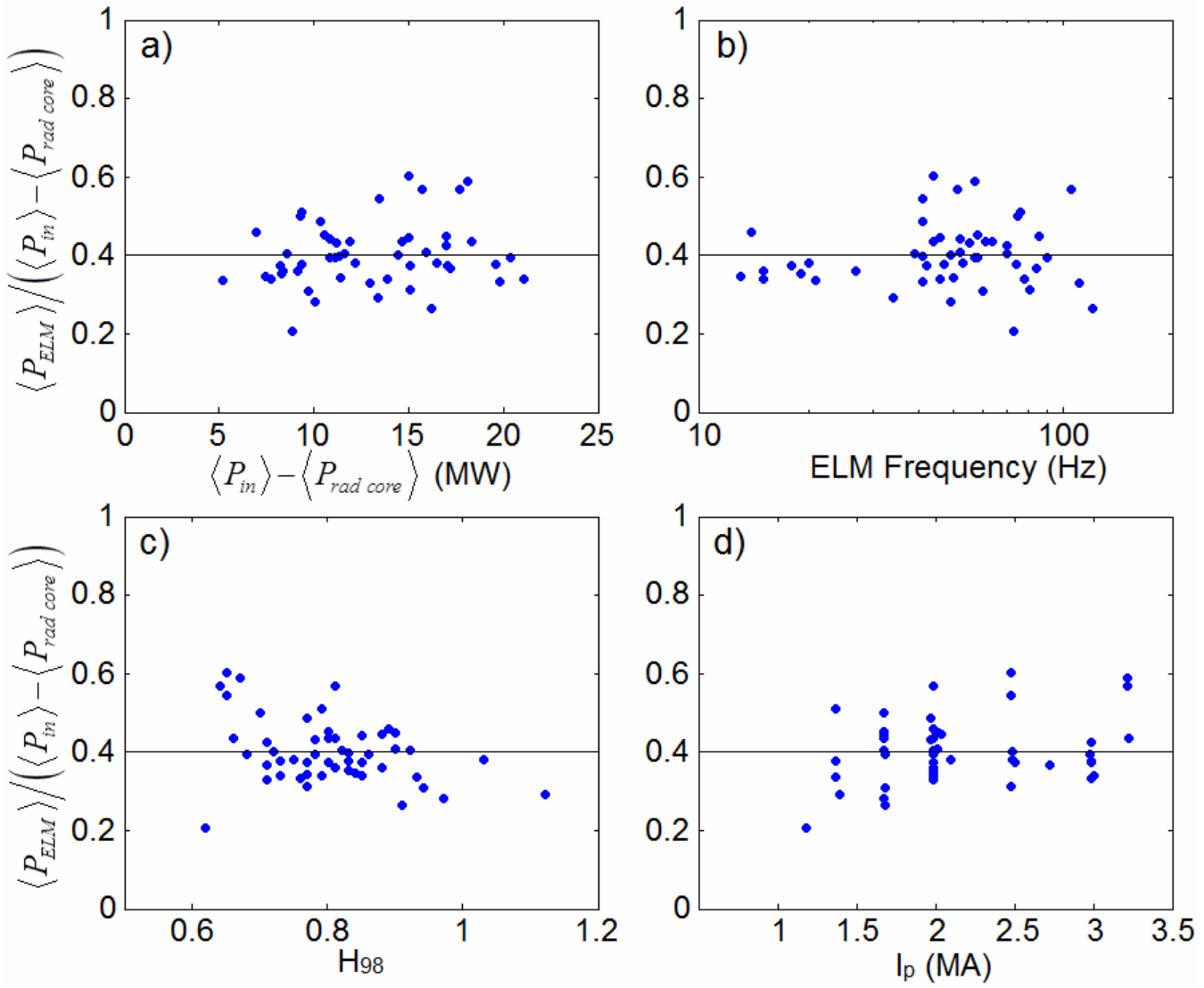

Fig. 4 


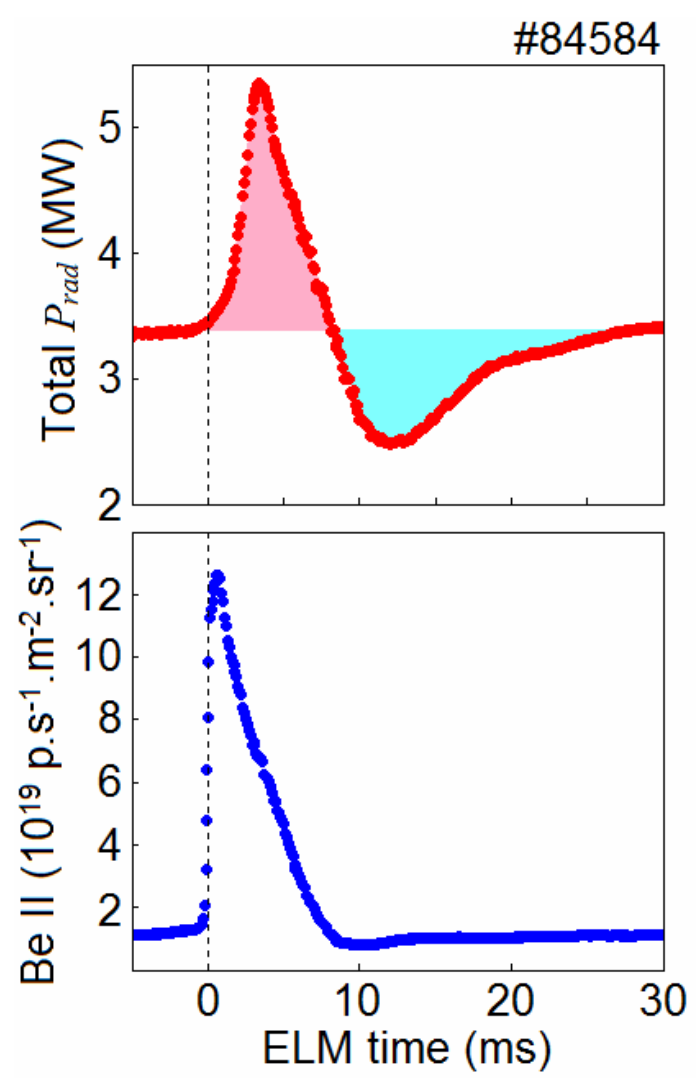

Fig. 5 


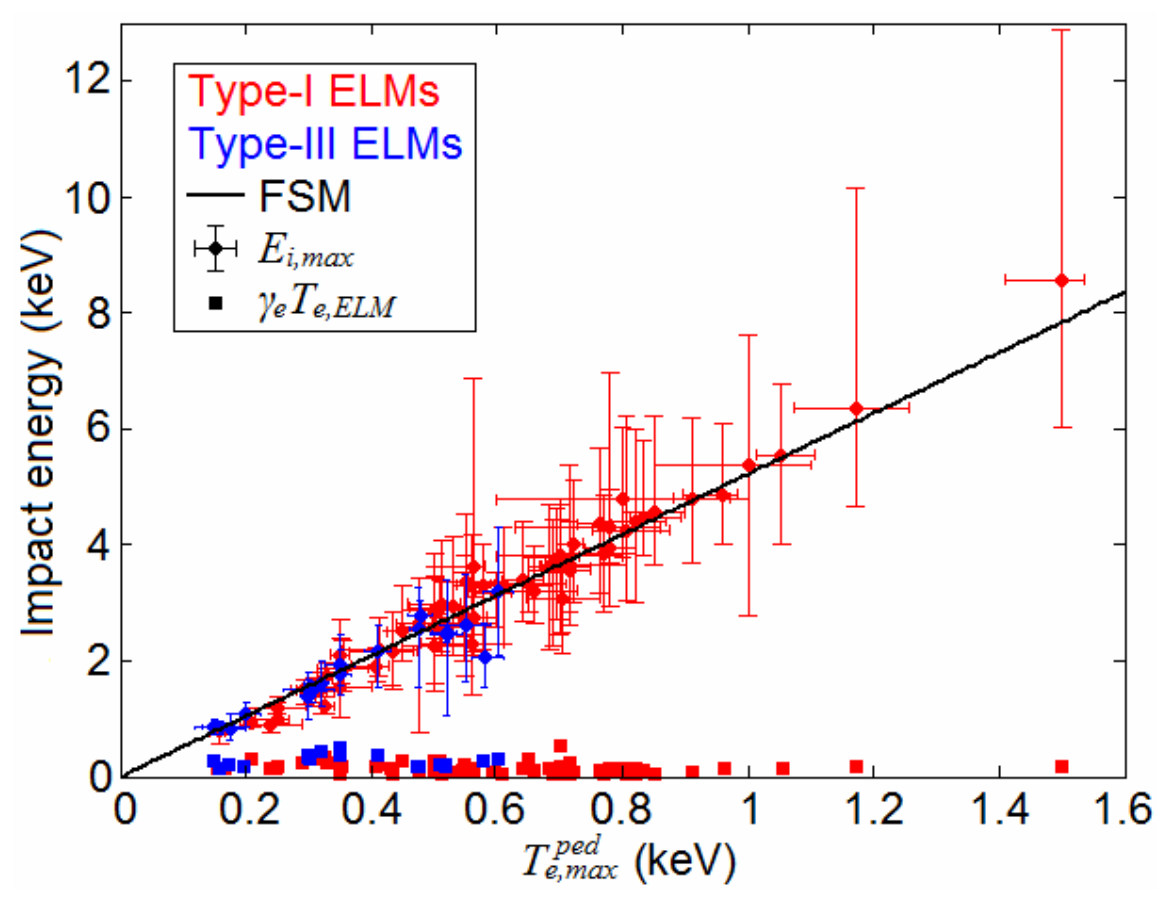

Fig. 6 